\title{
Preparation and characterisation of retrograded resistant starch
}

\author{
H. Patel ${ }^{1}$, G. Williams ${ }^{2}$, S. Gaisford ${ }^{2}$, D. McCarthy ${ }^{2}$, F. J Warren ${ }^{3}$, P. J Butterworth ${ }^{1}$ \\ and P. R. Ellis \\ ${ }^{1}$ School of Medicine, King's College London, SE1 9NH, ${ }^{2}$ School of Pharmacy, 29-39 Brunswick Square, University of \\ London, WCIN 1 AX and ${ }^{3}$ Centre for Nutrition and Food Sciences, Queensland Alliance for Agriculture and Food \\ Innovation, The University of Queensland, Australia
}

Starch digestion is of great importance to human health with a large fraction being digested in the small intestine. However, starch material that escapes digestion by amylolytic enzymes in the upper gastrointestinal tract is termed 'resistant starch' (RS). RS has been subdivided into four groups; physically inaccessible starch (RS1), native (uncooked) starch (RS2), retrograded (cooked and cooled) starch (RS3), and chemically modified starch (RS4). The amount of RS can vary considerably depending upon the botanical source and the food processing conditions applied. Many groups have reported that starches of high amylose content that have undergone temperature-cycled retrogradation contain increased amounts of retrograded starch (RS3) ${ }^{1}$. The purpose of this project is to harvest, characterise and perform enzyme inhibition studies on isolated RS from high amylose maize starch (HA).

In our study, retrogradation of HA at cycles of $4 / 37^{\circ} \mathrm{C}$ for 1 week significantly increased the RS content to $95 \%$. This allows accurate enzyme inhibition studies to be performed. X-ray diffraction (XRD), scanning electron microscopy (SEM) and nuclear magnetic resonance $\left({ }^{13} \mathrm{C}\right.$ NMR) was then used to determine the structure of RS. Surprisingly, upon XRD and NMR analysis, our results suggested that the isolated RS possessed low crystallinity. SEM images showed a web - like pattern connecting the starch material together. This resulted in associated 'lumps' of starch material rather than a continuous polymer network which is commonly seen in gelatinised starches. Thus the majority of the starch material is believed to be amorphous; however this amorphous material is clearly of a different form compared with the SEM images of amorphous gelatinised starches ${ }^{2}$. Upon digestion with pancreatic $\alpha$-amylase, no starch products were detected indicating RS is totally inert to digestion $(n=4)$. In addition to this, inhibition studies suggest RS acts as a non-competitive inhibitor of pancreatic $\alpha$-amylase.

To conclude, ready to eat chilled foods which have undergone retrogradation may have reduced starch digestibility. Therefore, the physiological effects of RS on human metabolism may have beneficial relevance in the management of health and disease (e.g. obesity and type II diabetes).

1. Zhang L, Hu X, Xu X et al. (2011) Carbohydr. Polym 84, 970-974.

2. Slaughter SL, Ellis PR, Jackson EC et al. (2002) Biochim Biophys Acta 1571, 55-63. 